\title{
Aqueous Self-Assembly of a Protein-Mimetic Ampholytic Block Copolypeptide
}

Jing Sun, ${ }^{\dagger \S}$ Peter Černoch, ${ }^{\ddagger \S}$ Antje Völkel, ${ }^{\S}$ Yuhan Wei, ${ }^{\dagger}$ Janne Ruokolainen, ${ }^{\|}$and Helmut Schlaad ${ }^{\perp \S}$

\footnotetext{
† School of Polymer Science and Engineering, Qingdao University of Science and Technology, 53 Zhengzhou Road, Qingdao 266042, China. ${ }^{\star}$ Institute of Macromolecular Chemistry, Heyrovského nám. 2, 16206 Praha 6, Czech Republic.

$\S$ Department of Colloid Chemistry, Max Planck Institute of Colloids and Interfaces, Research Campus Golm, 14424 Potsdam, Germany. ' Department of Applied Physics, Aalto University Nanomicroscopy Center (Aalto-NMC), Puumiehenkuja 2, 02150 Espoo, Finland, ${ }^{\perp}$ University of Potsdam, Institute of Chemistry, Karl-Liebknecht-Str. 24-25,
} 14476 Potsdam, Germany.

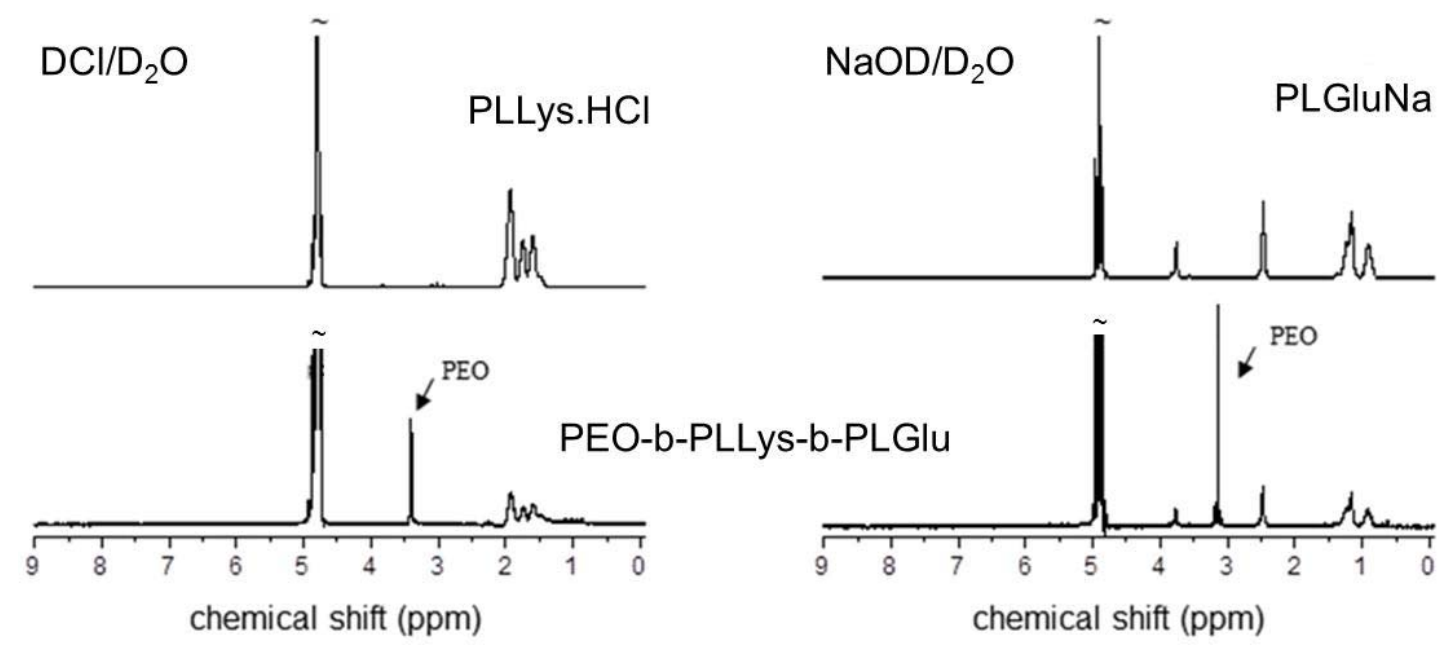

Figure S1. ${ }^{1} \mathrm{H}$ NMR spectra $\left(400.1 \mathrm{MHz}\right.$, r.t.) of PLLys and $\mathrm{PEO}_{42}-b-\mathrm{PLLys}_{61}-b-\mathrm{PLGlu}_{62}$ in $0.9 \mathrm{M} \mathrm{DCl}$ in $\mathrm{D}_{2} \mathrm{O}$ (left) and PLGlu and $\mathrm{PEO}_{42}-b$-PLLys ${ }_{61}-b-\mathrm{PLGlu}_{62}$ in $1.0 \mathrm{M} \mathrm{NaOD}$ in $\mathrm{D}_{2} \mathrm{O}$ (right).

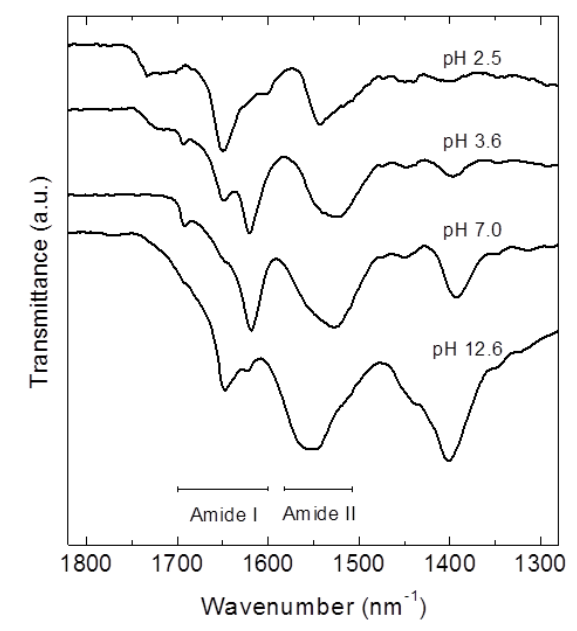

Figure S2. FT-IR spectra of freeze-dried samples of $0.2 \mathrm{wt} \% \mathrm{PEO}_{42}-\mathrm{PLLys}_{61}-\mathrm{PLGlu}_{62}$ in water at $\mathrm{pH} 2.5-12.6$. 


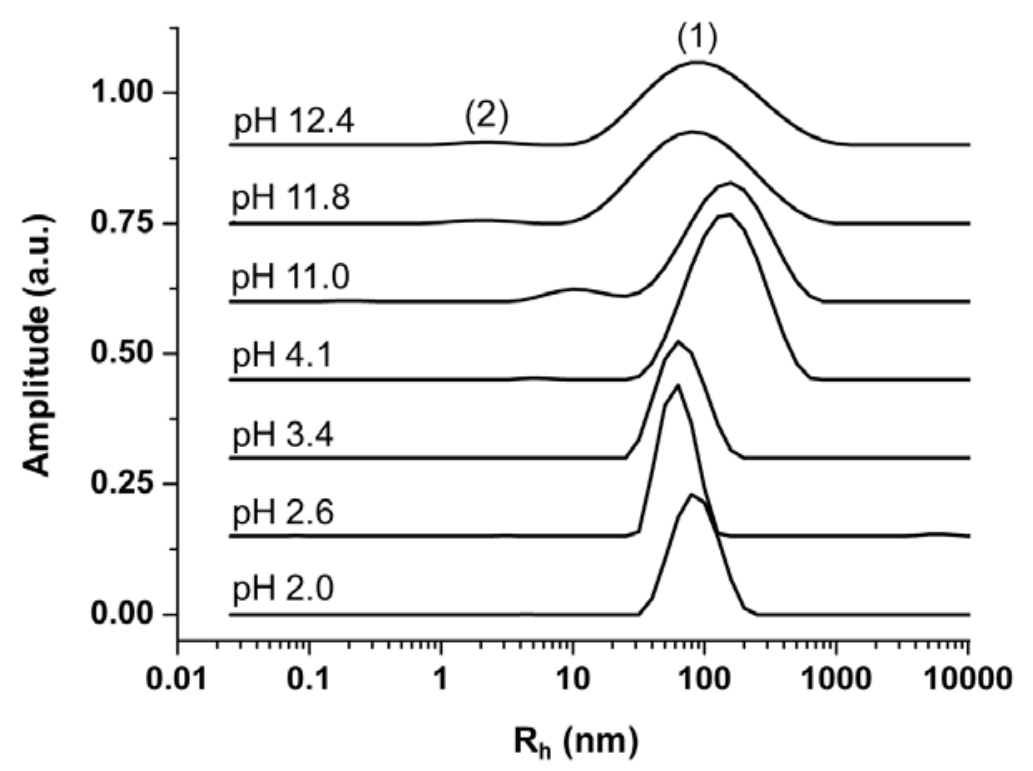

Figure S3. Normalized distributions of hydrodynamic radii $\left(R_{\mathrm{h}}\right)$ observed in $\sim 0.1 \mathrm{wt} \%$ solutions of $\mathrm{PEO}_{42}-b$-PLLys $61-b$-PLGlu 62 in $0.5 \mathrm{M} \mathrm{NaCl}$ at various $\mathrm{pH}$. Data measured at scattering angle $90^{\circ}$ at room temperature. (1) and (2) denote slow and fast modes, respectively.

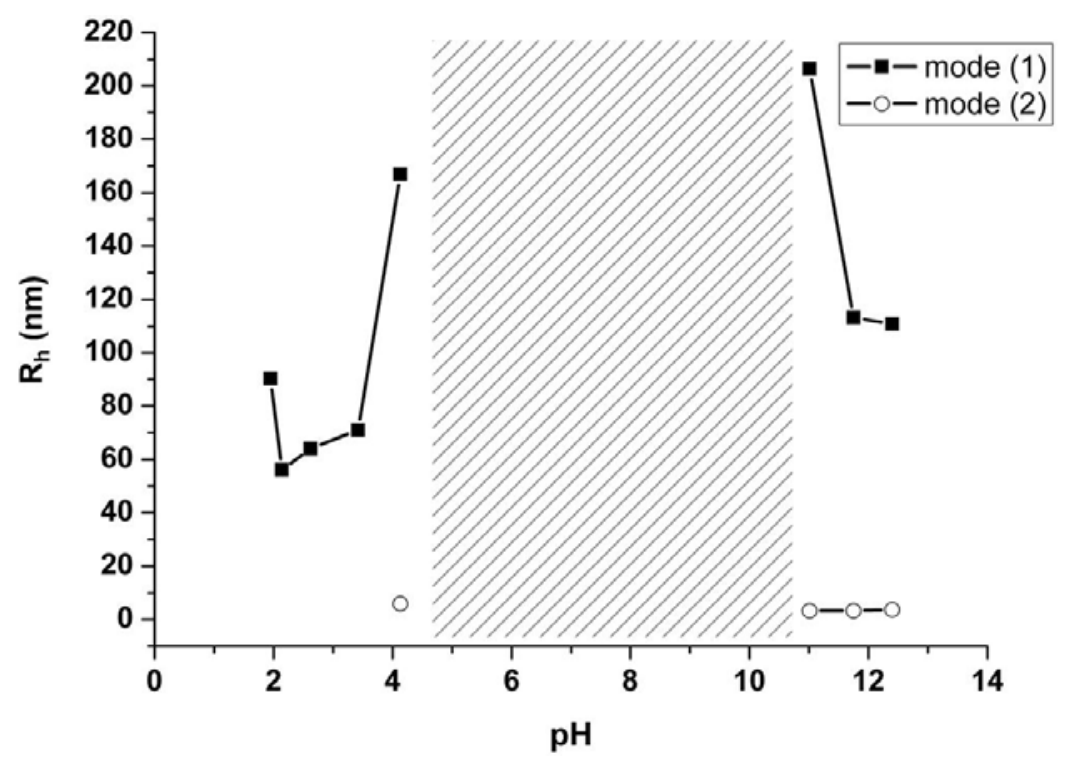

Figure S4. Dependence of hydrodynamic radii $\left(R_{\mathrm{h}}\right)$ observed in $\sim 0.1 \mathrm{wt} \%$ solutions of $\mathrm{PEO}_{42}-b$-PLLys $61-b$-PLGlu 62 in $0.5 \mathrm{M} \mathrm{NaCl}$ at various solution $\mathrm{pH}$. Data measured at scattering angle $90^{\circ}$ at room temperature. The hatched area represents an unstable region where all solutions demonstrate tendency to precipitate. Mode (1) is related to the major, slow diffusive mode of large aggregates, while mode (2) identifies the fast dynamic process of coupled diffusion of polyions and counterions or single chains. 


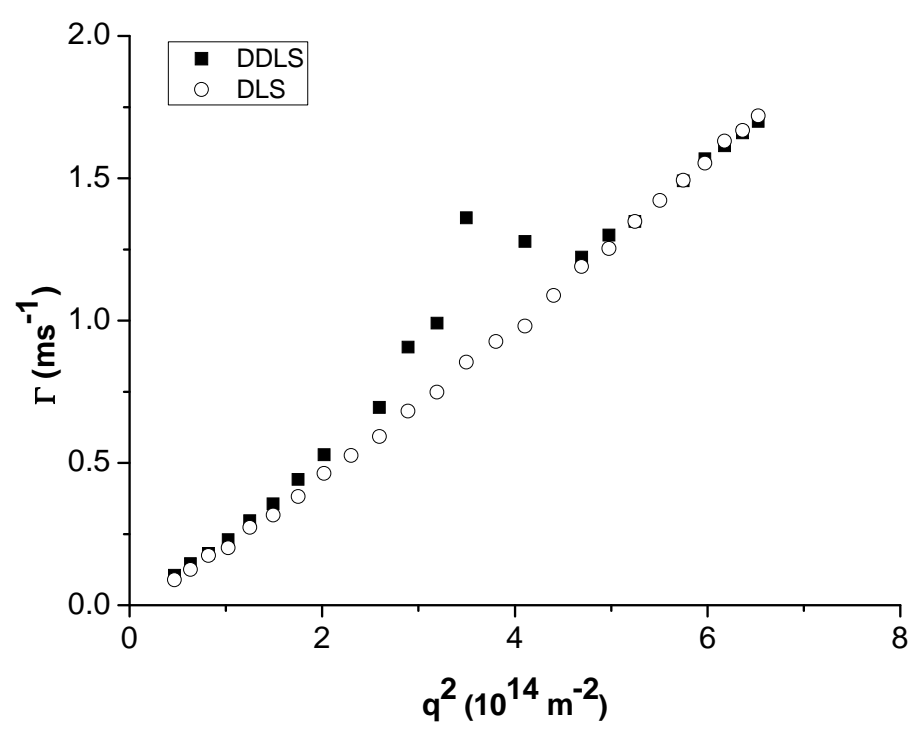

Figure S5. Relaxation rates $(\Gamma)$ obtained by DLS and DDLS as a function of the square of the scattering vector $\left(q^{2}\right)$ for the $\sim 0.1 \mathrm{wt} \%$ solutions of $\mathrm{PEO}_{42}-\mathrm{PLLys}_{61}-\mathrm{PLGlu}_{62}$ in $0.5 \mathrm{M}$ aqueous $\mathrm{NaCl}$ at $\mathrm{pH}$ 2.0.

DLS and DDLS gives linear dependence of decay rate with square of the scattering vector passing the origin $[0,0]$. The observable deviation around scattering angle of $\sim 90^{\circ}$ might be caused by either simultaneous presence of two diffusional effects with similar correlation times, which cannot be distinguished by the fitting algorithm, or deviation of the shape of the scattered objects from an ideal sphere. However, as there is not shift in the $\Gamma$-axis, which would indicate a contribution of rotational diffusion (Lehner et al. Langmuir 2000, 16, 1689-1695), the scattered objects are considered to be spherical in shape. 


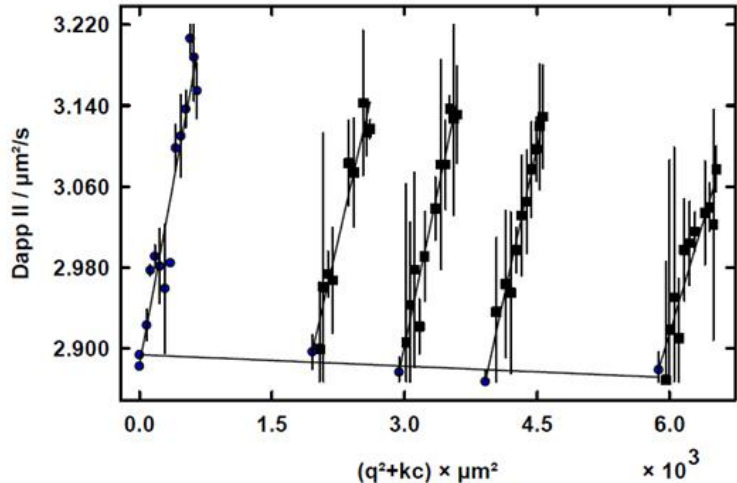

Dz(c): $2.894 \mathrm{e}+00 \mu \mathrm{m}^{2} / \mathrm{s} \quad \mathrm{Dz}\left(\mathrm{q}^{2}\right): 2.882 \mathrm{e}+00 \mu \mathrm{m}^{2} / \mathrm{s}$ $\mathrm{kD}:-5.492 \mathrm{e}-03 \mathrm{dm}^{2} / \mathrm{g} \quad \mathrm{C}<\mathrm{S}^{2}>1.635 \mathrm{e}-04 \mu \mathrm{m}^{2}$

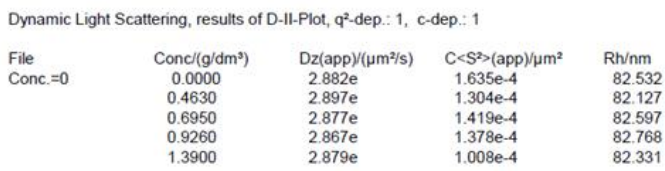

$\mathrm{Dz}(\mathrm{c}): 2.894 \mathrm{e}+00 \mu \mathrm{m}^{2} / \mathrm{s}( \pm 0.61 \%) \quad \mathrm{Dz}\left(\mathrm{q}^{2}\right): 2.882 \mathrm{e}+00 \mu \mathrm{m}^{2} / \mathrm{s}( \pm 0.85 \%)$ D. $5.492 \mathrm{e}-03 \mathrm{dm}^{2} / \mathrm{g}( \pm 119 \%) \quad \mathrm{C}<\mathrm{S}^{2} \mathrm{2} ; 1.635 \mathrm{e}-04 \mathrm{\mu m}^{2}( \pm 12.4 \%)$

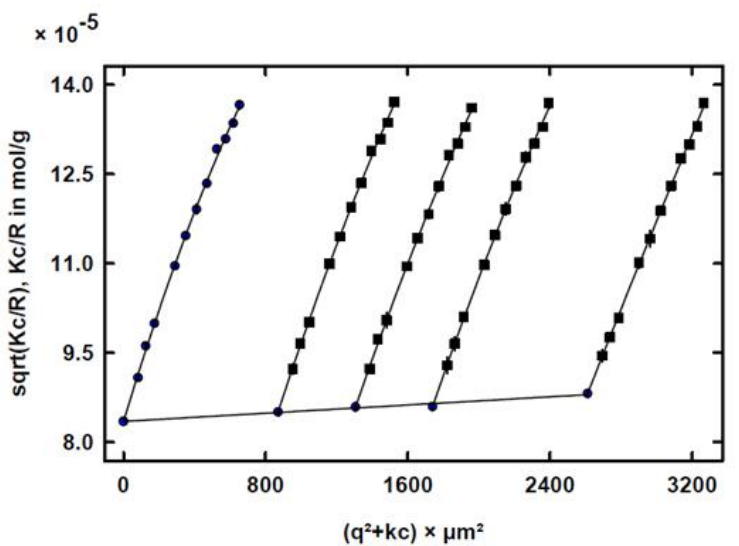

$M w(c): 1.435 \mathrm{e}+08 \mathrm{~g} / \mathrm{mol} \quad M w\left(q^{2}\right): 1.435 \mathrm{e}+08 \mathrm{~g} / \mathrm{mol}$ A2: $2.697 \mathrm{e}-10 \mathrm{~mol} \mathrm{dm} / \mathrm{g}^{2} \quad \mathrm{Rg}: 8.427 \mathrm{e}+01 \mathrm{~nm}$

Figure S6. Concentration- and angle-dependent DLS (left) and SLS (right) data for 0.05-0.14 $\mathrm{wt}^{\%} \% \mathrm{PEO}_{42}-\mathrm{PLLys}_{61}-\mathrm{PLGlu}_{62}$ in $0.5 \mathrm{M}$ aqueous $\mathrm{NaCl}$ at $\mathrm{pH} 2.0$ at room temperature.

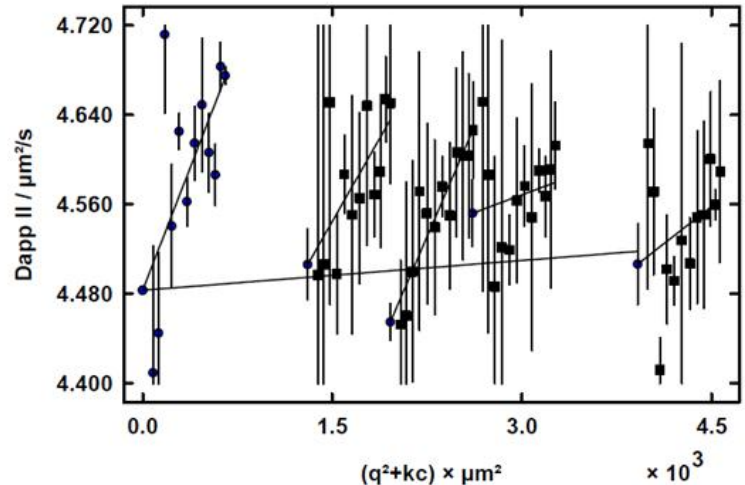

$D z(c): 4.483 \mathrm{e}+00 \mu \mathrm{m}^{2} / \mathrm{s} \quad$ Dz $\left(\mathrm{q}^{2}\right): 4.483 \mathrm{e}+00 \mu \mathrm{m}^{2} / \mathrm{s}$ kD: $5.184 \mathrm{e}-03 \mathrm{dm}^{3} / 9 \quad \mathrm{C}<\mathrm{S}^{2}>6.497 \mathrm{e}-05 \mu \mathrm{m}^{2}$
Dz(c): $4.483 \mathrm{e}+00 \mu \mathrm{m}^{2} / \mathrm{s}( \pm 1.43 \%) \quad \mathrm{Dz}\left(\mathrm{q}^{2}\right): 4.483 \mathrm{e}+00 \mu \mathrm{m}^{2} / \mathrm{s}( \pm 1.08 \%)$

Figure S7. Concentration- and angle-dependent DLS (left) and SLS (right) data for 0.05-0.15 wt $\% \mathrm{PEO}_{42}$-PLLys $61-\mathrm{PLGlu}_{62}$ in $0.5 \mathrm{M}$ aqueous $\mathrm{NaCl}$ at $\mathrm{pH} 2.2$ at room temperature. $\begin{array}{ll}M w(c)=5.958 \mathrm{e}+07 \mathrm{~g} / \mathrm{mol}( \pm 0.99 \%) & \mathrm{Mw}\left(\mathrm{q}^{2}\right): 5.958 \mathrm{e}+07 \mathrm{~g} / \mathrm{mol}( \pm 1.06 \%) \\ \mathrm{A} 2: 4.812 \mathrm{e}-10 \mathrm{~mol} \mathrm{dm} / \mathrm{g}^{2}( \pm 37.7 \%) & \mathrm{Rg}: 5.280 \mathrm{e}+01 \mathrm{~nm}( \pm 1.38 \%)\end{array}$

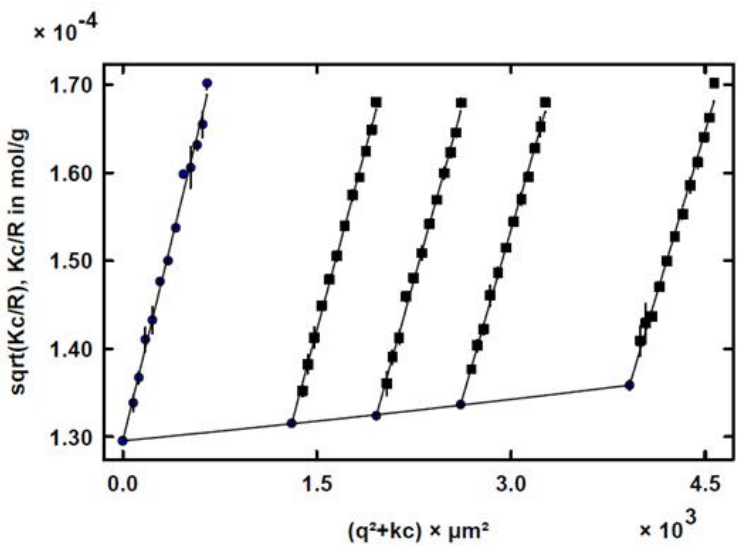

Mw(c): $5.958 \mathrm{e}+07 \mathrm{~g} / \mathrm{mol} \quad M w\left(q^{2}\right): 5.958 \mathrm{e}+07 \mathrm{~g} / \mathrm{mol}$ A2: $4.812 \mathrm{e}-10 \mathrm{~mol} \mathrm{dm} / \mathrm{g}^{2} \quad$ Rg: $5.280 \mathrm{e}+01 \mathrm{~nm}$

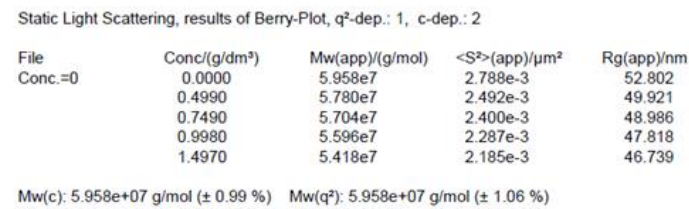

. 

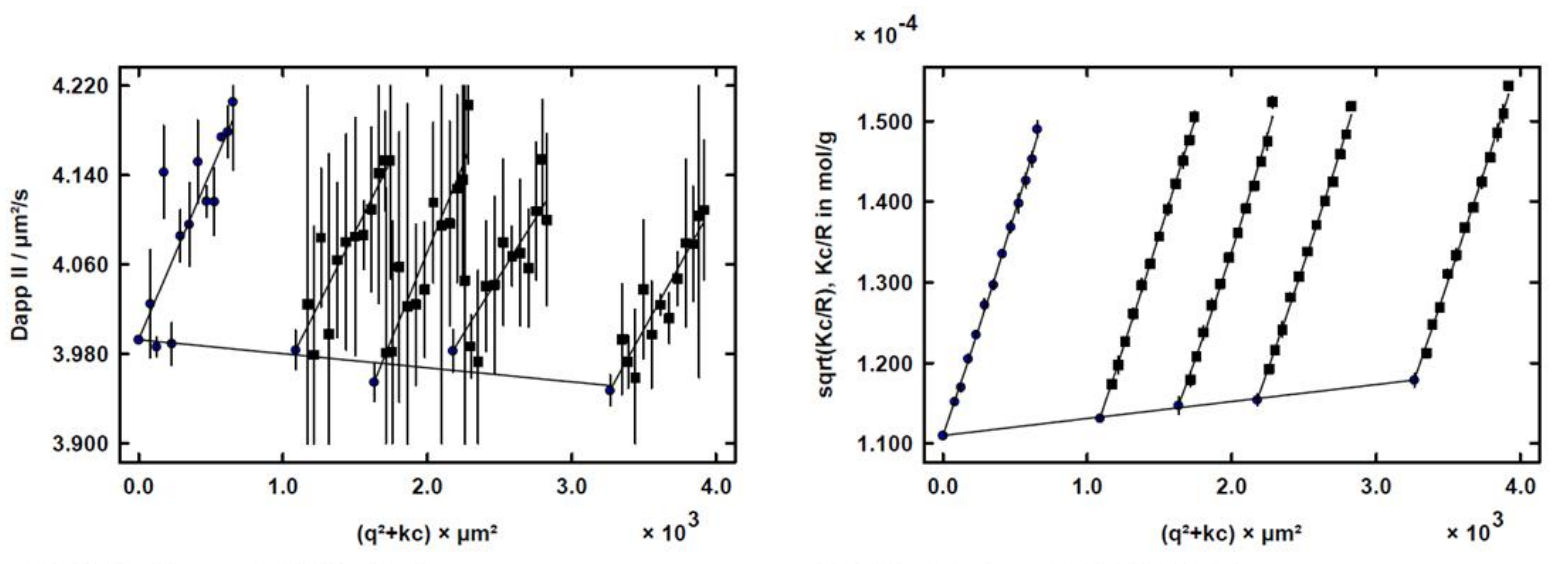

$\begin{array}{ll}\mathrm{Dz}(\mathrm{c}): 3.993 \mathrm{e}+00 \mu \mathrm{m}^{2} / \mathrm{s} & \mathrm{Dz}\left(\mathrm{q}^{2}\right): 3.993 \mathrm{e}+00 \mu \mathrm{m}^{2} / \mathrm{s} \\ \text { kD: }-7.219 \mathrm{e}-03 \mathrm{dm}^{3} / \mathrm{g} & \mathrm{C}<\mathrm{S}^{2}>7.540 \mathrm{e}-05 \mu \mathrm{m}^{2}\end{array}$

Mw(c): $8.118 \mathrm{e}+07 \mathrm{~g} / \mathrm{mol} \quad M w\left(\mathrm{q}^{2}\right): 8.118 \mathrm{e}+07 \mathrm{~g} / \mathrm{mol}$

$\begin{array}{ll}\text { A2: } 5.360 \mathrm{e}-10 \mathrm{~mol} \mathrm{dm} / \mathrm{g}^{2} & \text { Rg: } 5.273 \mathrm{e}+01 \mathrm{~nm}\end{array}$

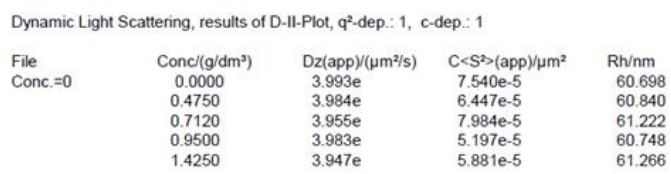

$D z(c): 3.993 e+00 \mu m^{2} / s( \pm 0.61 \%) \quad D z\left(q^{2}\right): 3.993 e+00 \mu m^{2} / s( \pm 0.72 \%)$

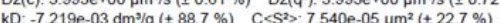

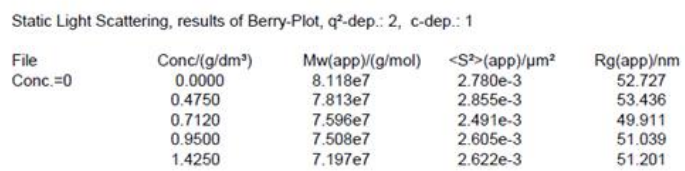

Mw(c): $8.118 \mathrm{e}+07 \mathrm{~g} / \mathrm{mol}( \pm 0.67 \%) \quad M w\left(q^{2}\right): 8.118 \mathrm{e}+07 \mathrm{~g} / \mathrm{mol}( \pm 1.2 \%)$

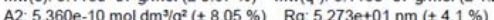

Figure S8. Concentration- and angle-dependent DLS (left) and SLS (right) data for 0.05-0.14 $\mathrm{wt}_{\%} \% \mathrm{PEO}_{42}-\mathrm{PLLys}_{61}-\mathrm{PLGlu}_{62}$ in $0.5 \mathrm{M}$ aqueous $\mathrm{NaCl}$ at $\mathrm{pH} 2.6$ at room temperature.

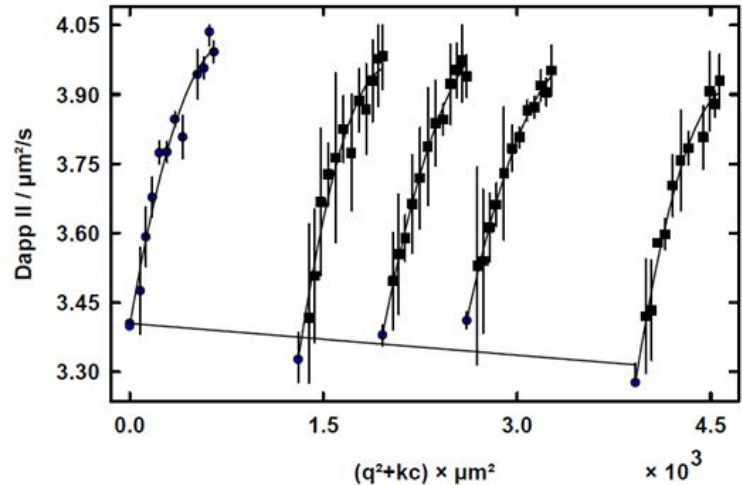

$\mathrm{Dz}(\mathrm{c}): 3.405 \mathrm{e}+00 \mu \mathrm{m}^{2} / \mathrm{s} \quad \mathrm{Dz}\left(\mathrm{q}^{2}\right): 3.400 \mathrm{e}+00 \mu \mathrm{m}^{2} / \mathrm{s}$ KD: $-2164 \mathrm{e}-02 \mathrm{dm}^{2} / 9 \quad \quad C<\mathrm{S}^{2}>4.747 \mathrm{e}-04 \mathrm{\mu m}^{2}$

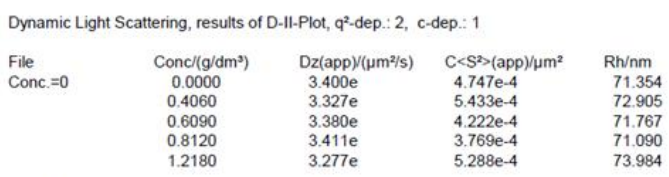

$\mathrm{Dz}(\mathrm{c}): 3.405 \mathrm{e}+00 \mu \mathrm{m}^{2} / \mathrm{s}( \pm 2.61 \%) \quad \mathrm{Dz}\left(\mathrm{q}^{2}\right): 3.400 \mathrm{e}+00 \mu \mathrm{m}^{2} / \mathrm{s}( \pm 1.58 \%)$ KD: $-2.164 \mathrm{e}-02 \mathrm{dm}^{2} / \mathrm{g}( \pm 147 \%) \quad \mathrm{C}<\mathrm{S}^{2}>-4.747 \mathrm{e}-04 \mu \mathrm{m}^{2}( \pm 21.5 \%)$

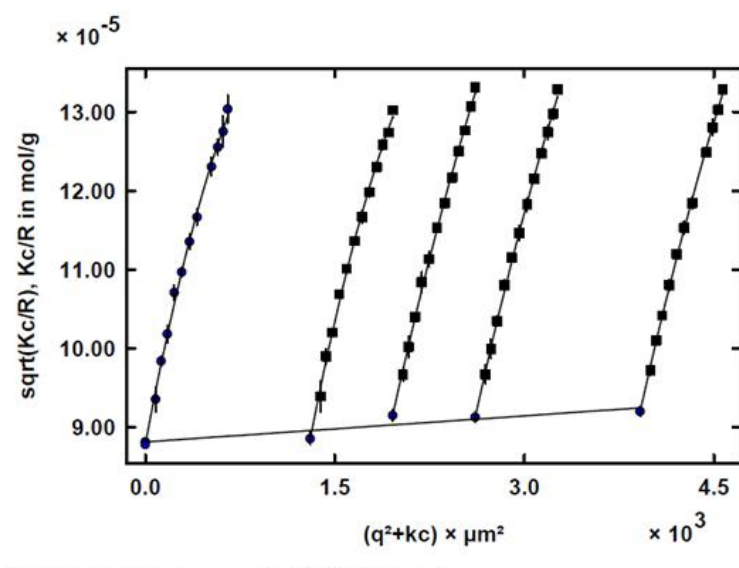

Mw(c): $1.286 \mathrm{e}+08 \mathrm{~g} / \mathrm{mol} \quad M w\left(q^{2}\right): 1.296 \mathrm{e}+08 \mathrm{~g} / \mathrm{mol}$ Rg: $7.630 \mathrm{e}+01 \mathrm{~nm}$

Figure S9. Concentration- and angle-dependent DLS (left) and SLS (right) data for 0.04-0.12 $\mathrm{wt}^{\mathrm{t}} \% \mathrm{PEO}_{42}-\mathrm{PLLys}_{61}-\mathrm{PLGlu}_{62}$ in $0.5 \mathrm{M}$ aqueous $\mathrm{NaCl}$ at $\mathrm{pH} 3.4$ at room temperature. 


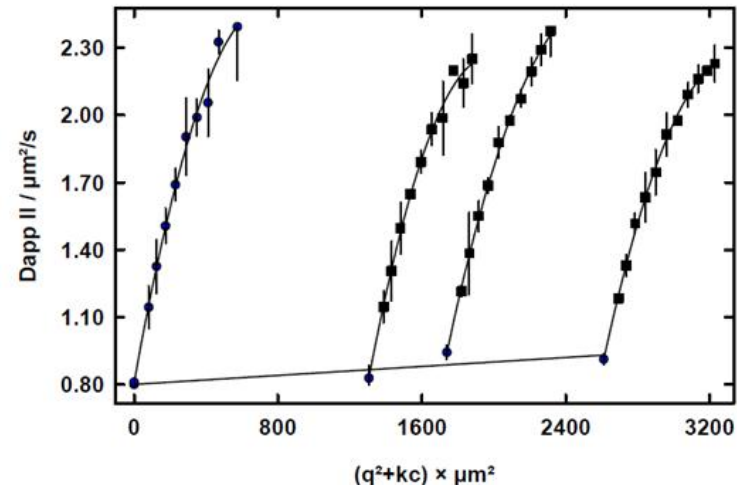

Dz(c): $8.001 \mathrm{e}-01 \mu \mathrm{m}^{2} / \mathrm{s} \quad$ Dz $\left(q^{2}\right): 8.104 \mathrm{e}-01 \mu \mathrm{m}^{2} / \mathrm{s}$ kD: $1.261 \mathrm{e}-01 \mathrm{dm}^{3} / 9 \quad C<\mathrm{S}^{2}>5.525 \mathrm{e}-03 \mu \mathrm{m}^{2}$

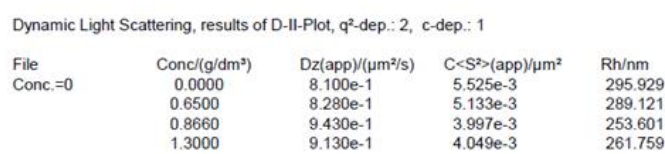
$\begin{array}{ll}\mathrm{Dz}(\mathrm{c}): 8.001 \mathrm{e}-01 \mu \mathrm{m}^{2} / \mathrm{s}( \pm 18.1 \%) & \mathrm{Dz}\left(\mathrm{q}^{2}\right): 8.104 \mathrm{e}-01 \mu \mathrm{m}^{2} / \mathrm{s}( \pm 8.78 \%) \\ \mathrm{kD}: 1261 \mathrm{e}-011 \mathrm{~m}^{2} / \mathrm{g}( \pm 148 \%) \quad C<\mathrm{S}^{2}>5.5525 \mathrm{e}-03 \mu \mathrm{m}^{2}( \pm 14.3 \%)\end{array}$

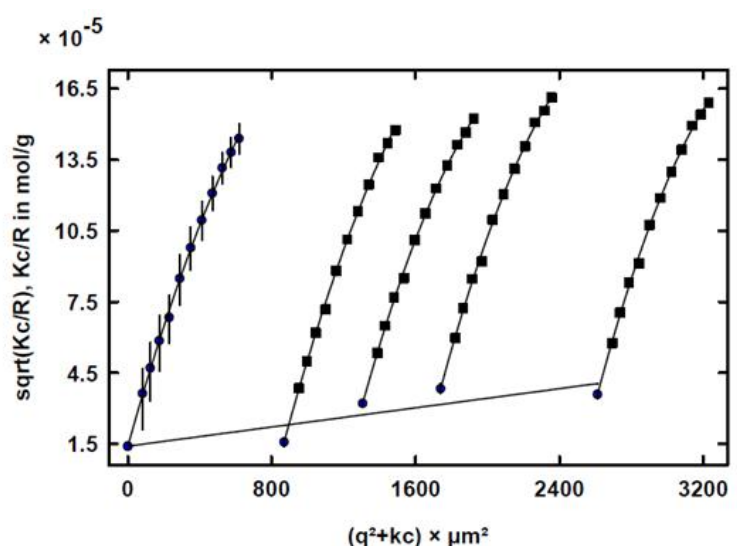

$M w(c): 5.046 \mathrm{e}+09 \mathrm{~g} / \mathrm{mol} \quad M w\left(q^{2}\right): 5.046 \mathrm{e}+09 \mathrm{~g} / \mathrm{mol}$ A2: $2.860 \mathrm{e}-10 \mathrm{~mol} \mathrm{dm} / \mathrm{g}^{2} \quad \mathrm{Rg}: 3.403 \mathrm{e}+02 \mathrm{~nm}$

Figure S10. Concentration- and angle-dependent DLS (left) and SLS (right) data for 0.04-0.13 wt $\% \mathrm{PEO}_{42}$-PLLys $61-\mathrm{PLGlu}_{62}$ in $0.5 \mathrm{M}$ aqueous $\mathrm{NaCl}$ at $\mathrm{pH} 4.1$ at room temperature.

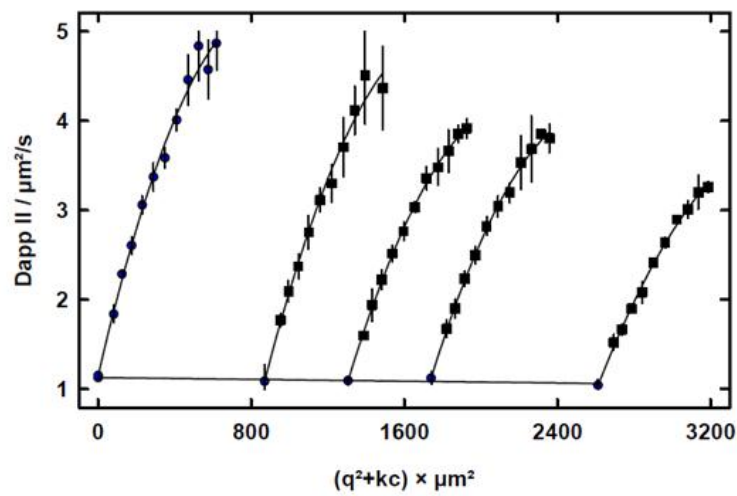

$\mathrm{Dz}(\mathrm{c}): 6.284 \mathrm{e}-01 \mu \mathrm{m} / \mathrm{s}$ kD: $-9.337 \mathrm{e}-02 \mathrm{dm}^{3 / 9} \quad$ Dz $\left(q^{2}\right): 6.510 \mathrm{e}-01 \mu \mathrm{m}^{2} / \mathrm{s}$ $\mathrm{C}<\mathrm{S}^{2}>1.420 \mathrm{e}-02 \mu \mathrm{m}^{2}$

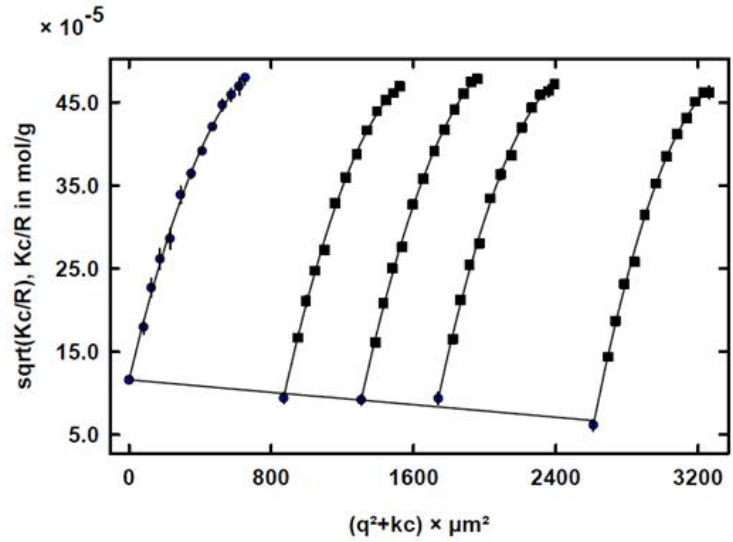

$M w(c): 7.419 \mathrm{e}+07 \mathrm{~g} / \mathrm{mol}$ A2: $-4.976 \mathrm{e}-09 \mathrm{~mol} \mathrm{dm}^{2} / \mathrm{g}^{2}$ $\operatorname{Mw}\left(q^{2}\right): 7.419 \mathrm{e}+07 \mathrm{~g} / \mathrm{mol}$ Rg: $2.152 \mathrm{e}+02 \mathrm{~nm}$
Dynamic Light Scattering, results of D-II-Plot, $q^{2}$-dep.: 2, c-dep.: 1

$\begin{array}{lcccc}\text { File } & \text { Conc/(g/dm }) & \mathrm{Dz}(\mathrm{app}) /\left(\mu \mathrm{m}^{2} / \mathrm{s}\right) & \mathrm{C}<\mathrm{S}^{2}>(\mathrm{app}) / \mu \mathrm{m}^{2} & \mathrm{Rh} / \mathrm{nm} \\ \text { Conc. }=0 & 0.0000 & 6.510 \mathrm{e}-1 & 1.420 \mathrm{e}-2 & 368.182 \\ & 0.3810 & 5.870 \mathrm{e}-1 & 1.416 \mathrm{e}-2 & 408.062 \\ & 0.5720 & 5.940 \mathrm{e}-1 & 1.181 \mathrm{e}-2 & 403.916 \\ & 0.7620 & 6.220 \mathrm{e}-1 & 1.112 \mathrm{e}-2 & 385.438 \\ & 1.1440 & 5.420 \mathrm{e}-1 & 1.008 \mathrm{e}-2 & 442.232\end{array}$

$\mathrm{Dz}(\mathrm{c}): 6.284 \mathrm{e}-01 \mu \mathrm{m}^{2} / \mathrm{s}( \pm 7.19 \%) \quad \mathrm{Dz}\left(\mathrm{q}^{2}\right): 6.510 \mathrm{e}-01 \mu \mathrm{m}^{2} / \mathrm{s}( \pm 27 \%)$ kD: $-9.337 \mathrm{e}-02 \mathrm{dm}^{3} / \mathrm{g}( \pm 100 \%) \quad \mathrm{C}<\mathrm{S}^{2}>1.420 \mathrm{e}-02 \mu \mathrm{m}^{2}( \pm 29.8 \%)$

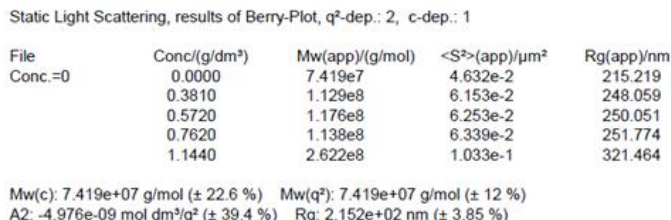

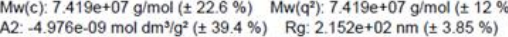

Figure S11. Concentration- and angle-dependent DLS (left) and SLS (right) data for 0.04-0.11 $\mathrm{wt}_{\%} \% \mathrm{PEO}_{42}-\mathrm{PLLys}_{61}-\mathrm{PLGlu}_{62}$ in $0.5 \mathrm{M}$ aqueous $\mathrm{NaCl}$ at $\mathrm{pH} 11.0$ at room temperature. 


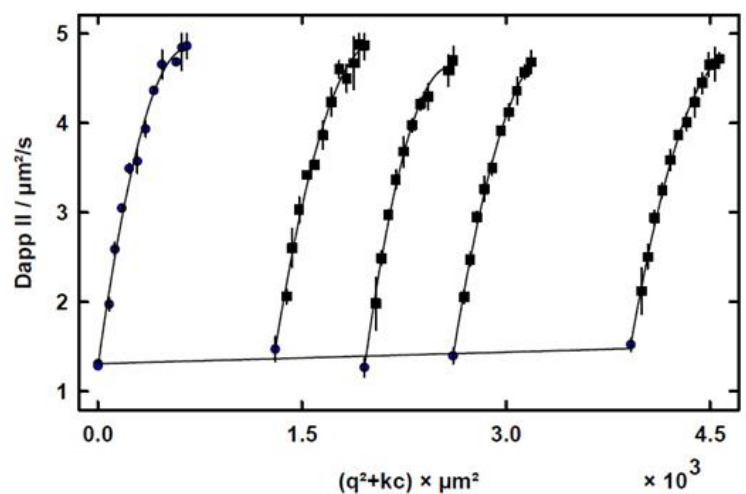

$D z(c): 1.308 \mathrm{e}+00 \mu \mathrm{m}^{2} / \mathrm{s} \quad$ Dz $\left(\mathrm{q}^{2}\right): 1.285 \mathrm{e}+00 \mu \mathrm{m}^{2} / \mathrm{s}$ kD: $1.131 \mathrm{e}-01 \mathrm{dm}^{2 / 9} \quad \mathrm{C}<\mathrm{S}^{2}>8.446 \mathrm{e}-03 \mu \mathrm{m}^{2}$

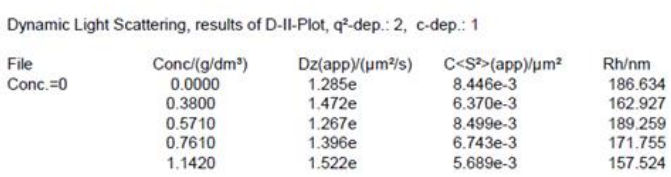

$\mathrm{Dz}(\mathrm{c}): 1.308 \mathrm{e}+00 \mu \mathrm{m}^{2} / \mathrm{s}( \pm 12.8 \%) \quad \mathrm{Dz}\left(\mathrm{q}^{2}\right): 1.285 \mathrm{e}+00 \mu \mathrm{m}^{2} / \mathrm{s}( \pm 12 \%)$ kD: $1.131 \mathrm{e}-01 \mathrm{dm}^{2} / \mathrm{g}( \pm 148 \%) \quad \mathrm{C}<\mathrm{S}^{2}>8.846 \mathrm{e}-03 \mu \mathrm{m}^{2}( \pm 15 \%)$

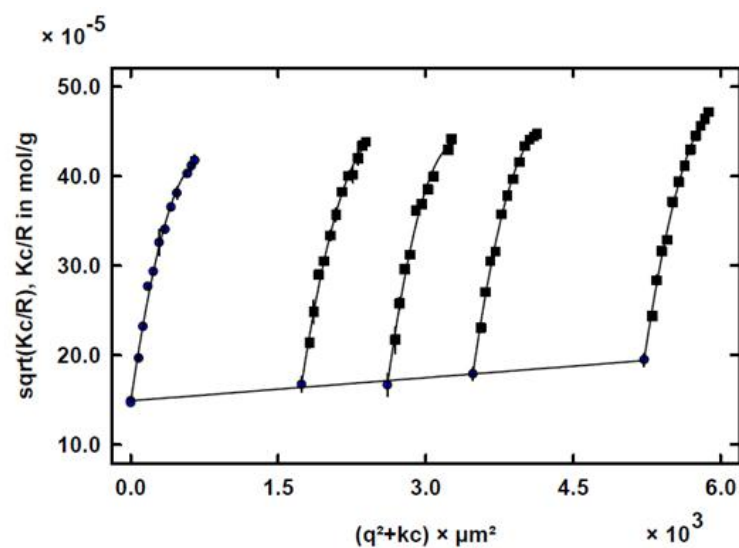

$\operatorname{Mw}(c): 4.505 \mathrm{e}+07 \mathrm{~g} / \mathrm{mol} \quad M w\left(q^{2}\right): 4.626 \mathrm{e}+07 \mathrm{~g} / \mathrm{mol}$ A2: $5.869 \mathrm{e}-09 \mathrm{~mol} \mathrm{dm} / \mathrm{g}^{2} \quad \mathrm{Rg}: 1.758 \mathrm{e}+02 \mathrm{~nm}$

Figure S12. Concentration- and angle-dependent DLS (left) and SLS (right) data for 0.04-0.11 wt $\% \mathrm{PEO}_{42}$-PLLys 61 -PLGlu 62 in $0.5 \mathrm{M}$ aqueous $\mathrm{NaCl}$ at $\mathrm{pH} 11.8$ at room temperature.

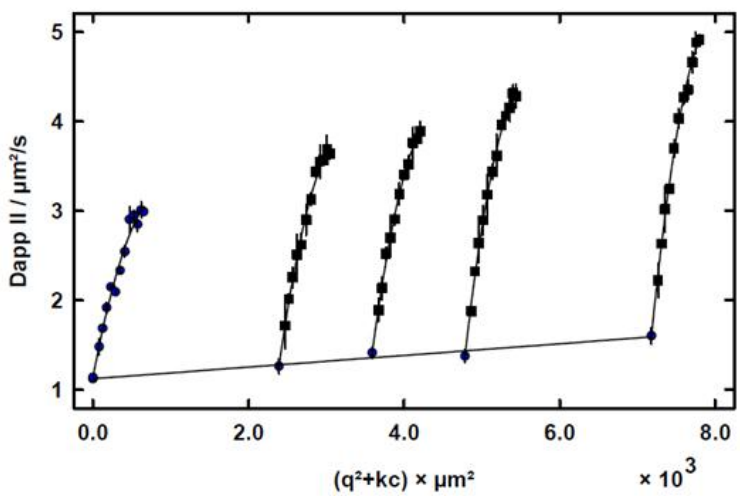

$D z(c): 1.123 \mathrm{e}+00 \mu \mathrm{m}^{2} / \mathrm{s} \quad \mathrm{Dz}\left(\mathrm{q}^{2}\right): 1.138 \mathrm{e}+00 \mu \mathrm{m}^{2} / \mathrm{s}$ $\mathrm{kD}: 3.462 \mathrm{e}-01 \mathrm{dm}^{2} / \mathrm{g} \quad \mathrm{C}<\mathrm{S}^{2}>4.055 \mathrm{e}-03 \mu \mathrm{m}^{2}$

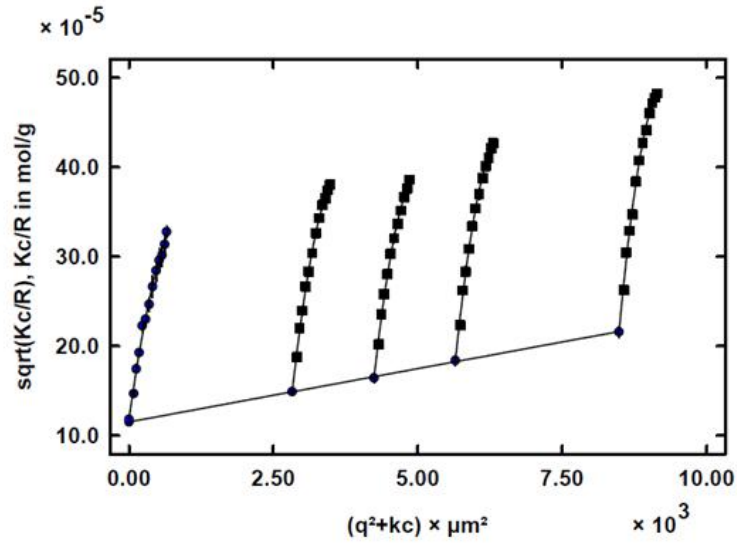

$M w(c): 7.553 \mathrm{e}+07 \mathrm{~g} / \mathrm{mol}$ A2: $9.672 \mathrm{e}-09 \mathrm{~mol} \mathrm{dm} / \mathrm{g}^{2}$ $\mathrm{Rg}: 1.525 \mathrm{e}+02 \mathrm{~nm}$

Static Light Scattering, results of Berry-Plot, $\mathrm{q}^{2}$-dep.: 2, c-dep.: 1

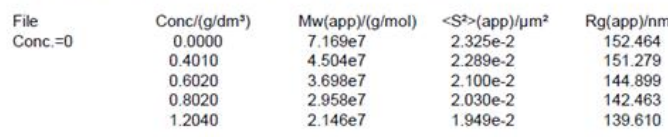

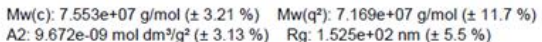

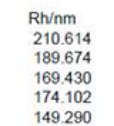

Figure S13. Concentration- and angle-dependent DLS (left) and SLS (right) data for 0.04-0.12 ${ }_{\mathrm{wt}} \% \mathrm{PEO}_{42}$ - $\mathrm{PLLys}_{61}-\mathrm{PLGlu}_{62}$ in $0.5 \mathrm{M}$ aqueous $\mathrm{NaCl}$ at $\mathrm{pH} 12.4$ at room temperature. 

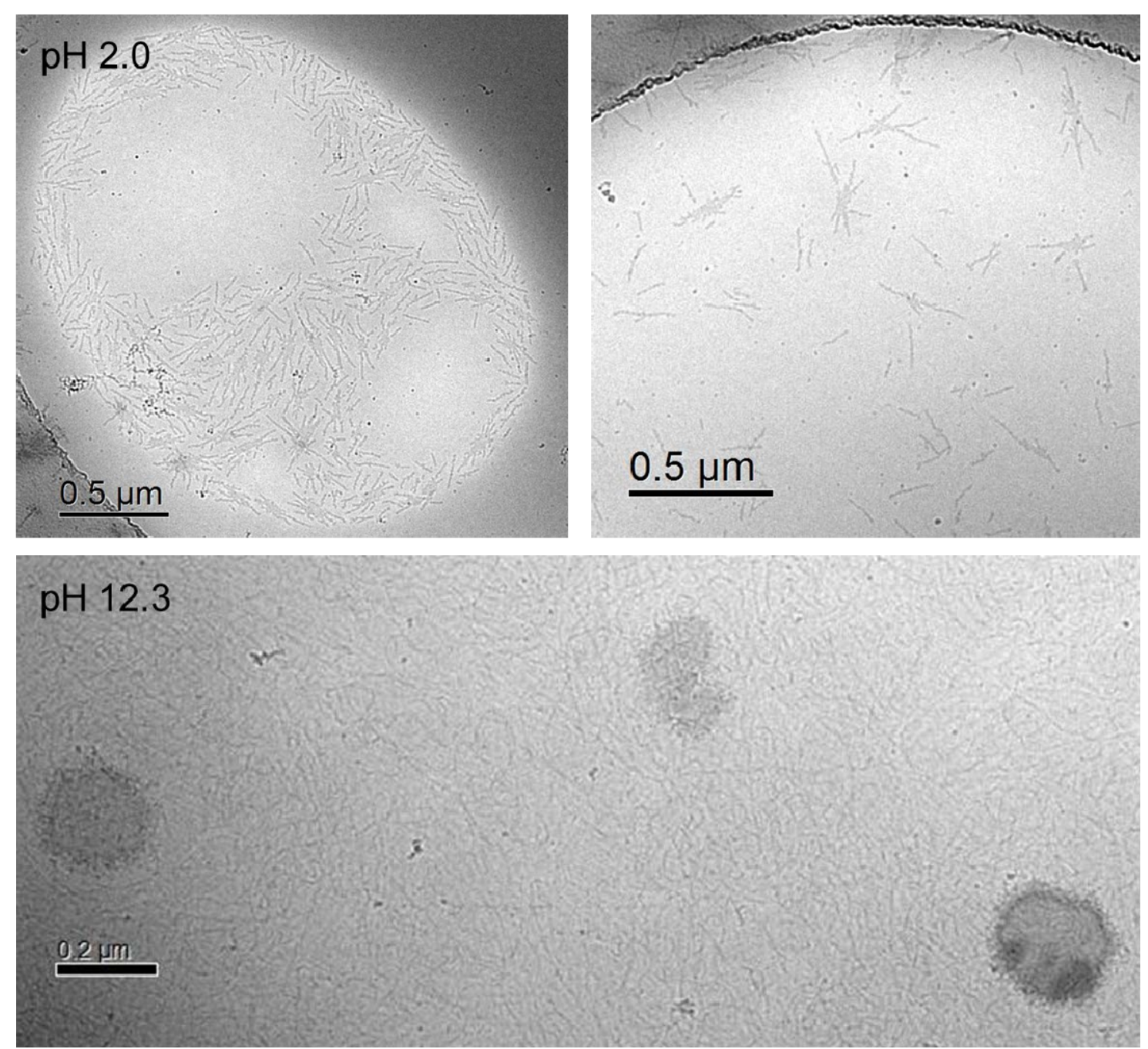

Figure S14. Exemplary cryogenic transmission electron micrographs of aged $\sim 0.1 \mathrm{wt} \%$ solutions of $\mathrm{PEO}_{42}-b$-PLLys $61-b$ - $\mathrm{PLGlu}_{62}$ in $0.5 \mathrm{M} \mathrm{NaCl}$ at $\mathrm{pH} 2.0$ (top) and $\mathrm{pH} 12.4$ (bottom), showing either discrete rod-like structures or vesicle-like particles and fibers (instead of vesicles or no aggregates); samples were analyzed $\sim 10$ months after their preparation.

Experimental procedure. $3 \mu \mathrm{L}$ of sample solution was applied on plasma-treated (Gatan Solarus Model 950 Advanced Plasma System, $p=70$ mTorr, $\mathrm{H}_{2}$ flow $6.4 \mathrm{sccm}, \mathrm{O}_{2}$ flow 27.5 sccm, forward RF target $50 \mathrm{~W}$, time $30 \mathrm{~s}$ ) carbon copper grids (Quantifoil R 3.5/1) in the environmental chamber of FEI Vitrobot having relative air humidity of $100 \%$ and temperature of $22{ }^{\circ} \mathrm{C}$. The excess solution was removed by blotting with filter paper for $1.5-2$ seconds followed by $0-30$ seconds draining and plunging of the samples into 1:1 mixture of liquid ethane and liquid propane, which was cooled below $-170{ }^{\circ} \mathrm{C}$. Vitrified samples were cryo-transferred into Jeol JEM-3200FSC cryo-transmission electron microscope operating at $-194{ }^{\circ} \mathrm{C}$. The temperature of the samples was $-187{ }^{\circ} \mathrm{C}$ during the imaging. The microscope was operating in the bright field mode, using $300 \mathrm{kV}$ acceleration voltage and the in-column energy filter set to $0-20 \mathrm{eV}$ energy-loss range (zero-loss imaging). Micrographs were recorded with Gatan Ultrascan 4000 CCD camera. 

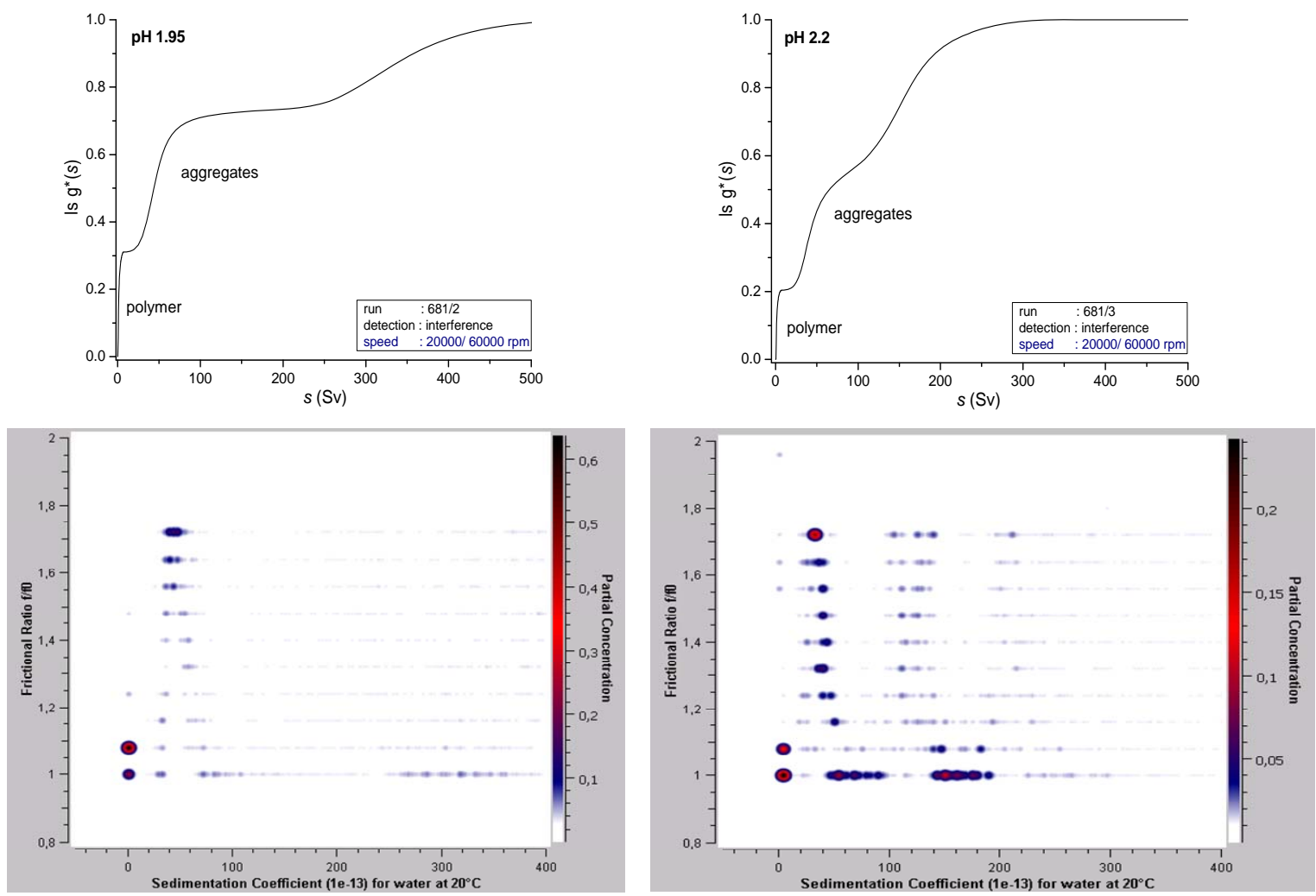

Figure S15. AUC sedimentation coefficient distributions (top) and pseudo 3D plots (UltraScan 2DSA-MC) of frictional ratio $\left(f / f_{\mathrm{o}}\right) v s$. sedimentation coefficient (bottom) of aged $\sim 0.1 \mathrm{wt} \%$ solutions of $\mathrm{PEO}_{42}-b$-PLLys $61-b-\mathrm{PLGlu}_{62}$ in $0.5 \mathrm{M} \mathrm{NaCl}$ at $\mathrm{pH} 2.0$ and $\mathrm{pH} 2.2$; samples were analyzed $\sim 3$ months after their preparation.

Lamm equation fitting with 2DSA-MC analysis. Sedimentation and diffusion terms are used to generate solutions for the direct modeling of the sedimentation boundary, using the two dimensional spectrum analysis (2DSA) implemented in the software package UltraScan III. The frictional ratio $\left(f f f_{0}\right)$, which gives a hint to the morphology of the sedimenting species, is determined with a model-free grid search followed by a Monte Carlo (MC) approach. It can be plotted as a function of the sedimentation coefficient in a pseudo 3D graph where the concentration of each species builds the pseudo z-axis (E. Brookes, W. Cao, B. Demeler, Eur. Biophys. J. 2010, 39, 405). Values of $f / f_{0} \sim 1$ indicate structures with globular (spherical) shape whereas $f l f_{0} \sim>1$ indicate non-globular structures (e.g., rods) (K. E. van Holde, W. C. Johnson, P. S. Ho, Principles of PhysicalBiochemistry, $2^{\text {nd }}$ edition, Intl. ed., Prentice-Hall, EnglewoodCliffs, NJ 2005; C. R. Cantor, P. R. Schimmel, Biophysical Chemistry, Freeman Part II, San Francisco, CA 1980.). Exact dimensions of aggregates could not be determined because of different specific volumes of polymer chains and aggregates.

Calculations were performed on the UltraScan LIMS cluster at the Bioinformatics Core Facility at the University of Texas Health Science Center at San Antonio and XSEDE resources supported by NSF XSEDE Grant \#MCB070038 (to Borries Demeler), and the Gateway is made possible by the use of XSEDE resources and Extended Collaborative Support Service (ECSS) Program funded by the NSF through the award OCI-1053575. 